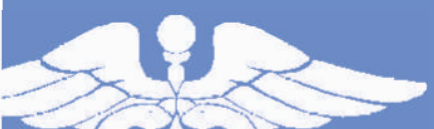

ISSN: 2782-7550 (Print) ISSN: 2782-7542 (Online)
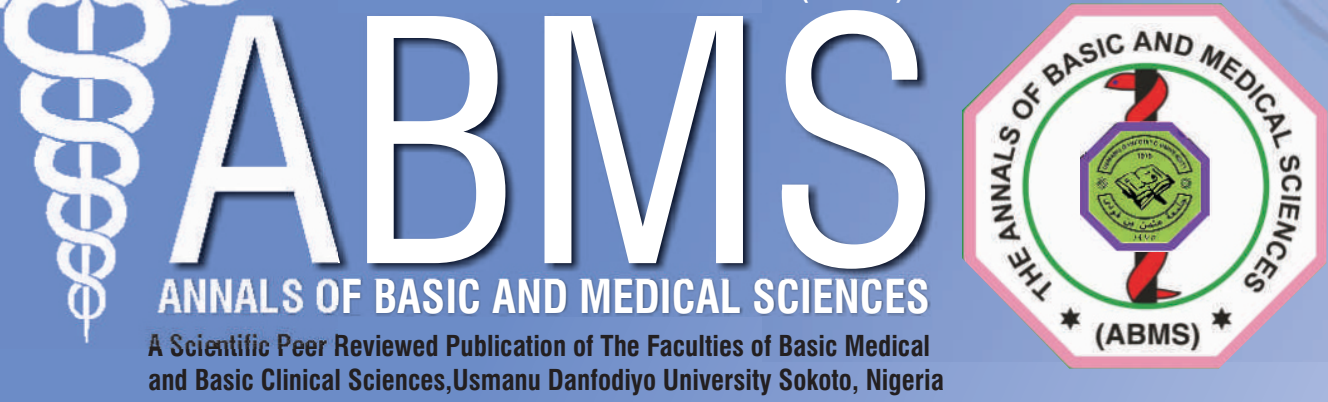

and Basic Clinical Sciences,Usmanu Danfodiyo University Sokoto, Nigeria
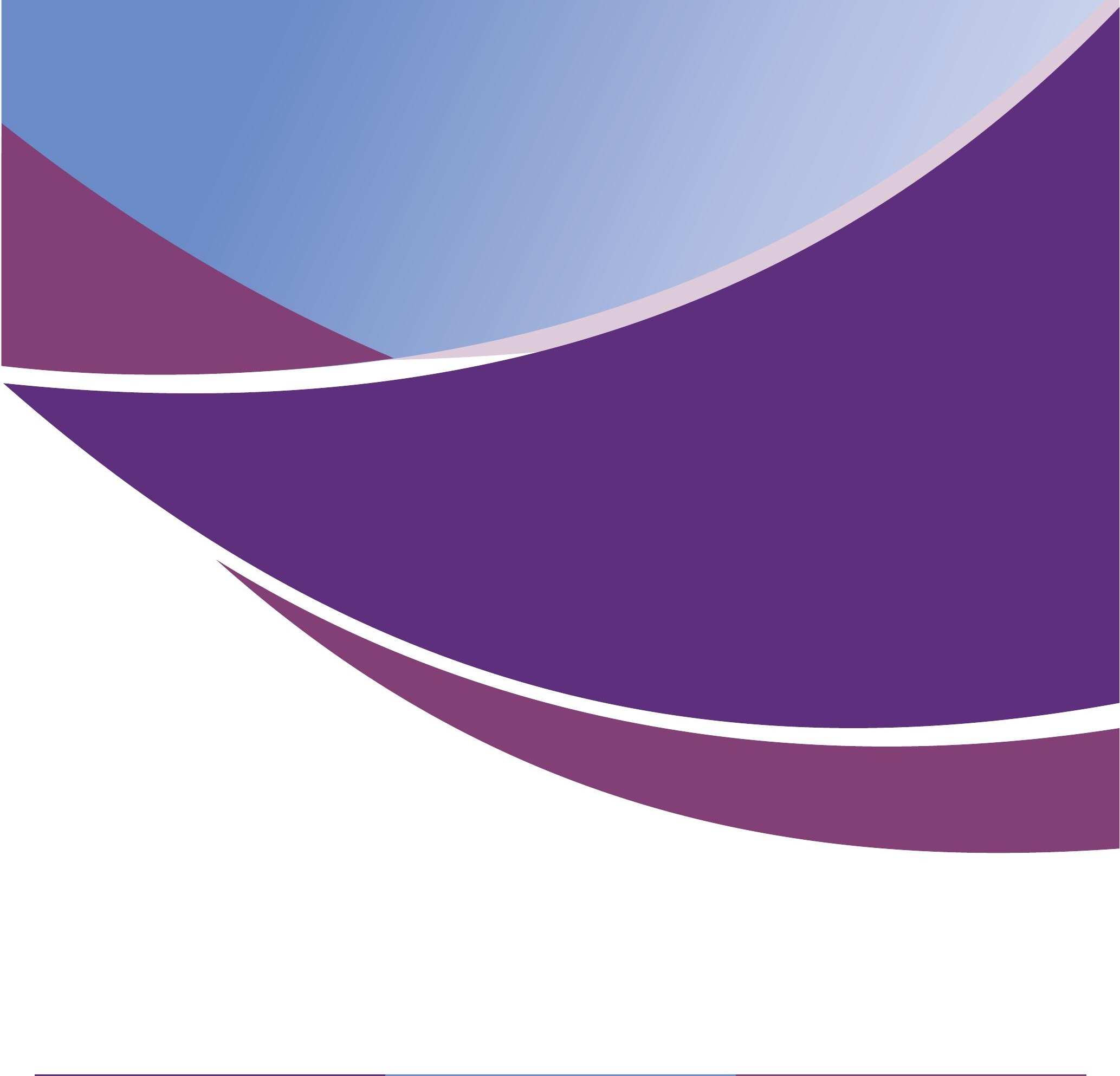

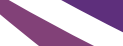
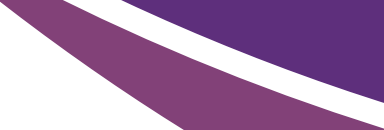


\title{
A Rare Case of Generalized Tetanus Complicating Breast Cancer: A Consequence of Harmful Cultural Practices.
}

\author{
Garba D. Waziri', Mukhtar A. Adeiza', Ibrahim U. Toli', Emmanuel Iwuozo ${ }^{3}$ \\ 'Department of Pathology, Ahmadu Bello University and Teaching Hospital, Shika Zaria \\ ${ }^{2}$ Infectious diseases/Respiratory Unit, Department of Medicine, Ahmadu Bello University and Teaching Hospital, Shika Zaria \\ ${ }^{3}$ Neurology Unit, Department of Medicine, Ahmadu Bello University and Teaching Hospital, Shika Zaria
}

\begin{abstract}
:
Tetanus infection is a public health disease and is still a cause of high morbidity and mortality in developing countries compared to the developed countries of the world. Apart from the traditional risk factors for tetanus, ignorance and harmful cultural practices greatly increase the risk and poor outcomes of tetanus in our country. We report this case of fatal generalized tetanus infection from necrotic fungating tumour following application of traditional herbal medication to highlight the link between a non-communicable disease, an infectious disease and poor health seeking behaviour in a global health context. We also reviewed similar cases reported in the literature. Patient consent was sought for before her death and patient confidentiality was assured.
\end{abstract}

Key words: tetanus, breast cancer, cultural practice, vaccination.

Corresponding author: Dr Garba D. Waziri

Department of Pathology, Ahmadu Bello University and Teaching Hospital, Shika Zaria wazirdg@gmail.com $+2348036268972$
Introduction

7 etanus is a vaccine preventable life threatening neurologic disease. It is an endemic infection especially in the developing countries largely due to inadequate vaccination uptake (1) poor environmental sanitation (2), suboptimal storage facilities for vaccines, ignorance and harmful cultural practices (3). Cancer patients are particularly vulnerable to tetanus infection because of immuno-compromised state, advance age, chronic psychological stress and use of immunosuppressive drugs $(2,4)$. In this case, we report a fatal case of generalized tetanus following use of topical traditional medication on a fungating breast cancer. This raises issues of adequate health education, vaccination and timely treatment of tetanus in advanced breast cancer.

\section{Case Report}

Mrs A.M. was a 73 year old widow that presented to the accident and emergency on 27/03/2013 with one day history of inability to open mouth and 7 hour history of repeated generalised spasms; she had a total of 14 unprovoked and 6 provoked spasms. There was associated difficulty in swallowing and body pains. No history of fever, sweating, palpitations, convulsion or loss of consciousness. She was diagnosed with histology confirmed right breast cancer at stage IV in 2012. She however declined orthodox management and opted for traditional medications despite serial counselling sessions. The skin over the tumour was initially intact, but it was later breached from multiple traditional interventions. A topical powdery mixture was applied to the ulcerated tumour. There was no history of any other injuries or co-morbidity. She had no prior history of tetanus vaccination despite being multiparous. Physical examination findings revealed a wasted elderly woman that was fully conscious, pale with enlarged right axillary lymph nodesthat were hard in consistency and fixed to the underlying tissues. She was 1.7 meters tall and weighed 50 kilograms with a BMI of about $17.3 \mathrm{Kg} / \mathrm{m}^{2}$. There was trismus, nuchal rigidity with neck retraction and opisthotonus. There were no focal neurological deficits. She was tachypneic with regular respiratory rate of 32 cycles per minute with vesicular breath sounds of reduced intensity and bilateral infra axillary crackles. Pulse was regular with rate of 110 beat per minute, blood pressure was $120 / 80 \mathrm{mmHg}$ and normal heart sounds. There was a hard fixed lump in the upper outer quadrant that extended to the upper inner quadrant of the right breast. The lump measured $12 \mathrm{~cm}$ by $10 \mathrm{~cm}$ with areas of redness and swelling. There was an ulcer at the medial aspect of the mass that measured $6 \mathrm{~cm}$ by $6 \mathrm{~cm}$ with everted edge, necrotic floor with bloody, purulent discharge. The left breast, abdominal and musculoskeletal systems examination findings were normal. She was admitted as a case of generalised tetanus grade III using Ablett classification[5] with superimposed sepsis and managed as per tetanus treatment protocol. She received parenteral anti tetanus serum (10,000i.u), tetanus toxoid, Metronidazole (500mg tds), Diazepam infusion (120mg/24 hours in glucose containing IV fluid to be titrated as 
titrated as required), Ceftriazone and supportive care that included oxygen therapy, nasogastric tube for feeding, prevention of deep vein thrombosis and bed sores. She also had breast wound debridement done. Patient passed away on 28/3/2013after admission. Available investigations revealed a histological diagnosis of invasive ductal carcinoma NOS, Nothingham grade III. Wound culture yielded a polymicrobial growth but there was no facility for anaerobic culture at that time.

\section{Discussion}

Tetanus is a fatal neurological disease with potentially high morbidity and mortality especially among certain group of patients like the elderly and cancer patients. This group has waning immunity and thus low levels of neutralising immunoglobulins due to advanced age, malignancy and use of immunosupressive chemotherapy (1). Tetanus is caused by the gram positive anaerobic bacteria Clostridium tetani. Clinical features of the disease are based on neurotoxin mediated actions of tetanospasmin that is produced by the organisms (6). Nigeria has a high burden of breast cancer with more than 500,000 new reported cases every year characterised by late presentation (7). There are few reported cases of tetanus among advanced breast cancer patients and therefore no available data on its incidence among this group of patients (4). This may be due to rarity of tetanus in developed countries because of vaccination or under reportage in poor resource nations. In our setting, tetanus is endemic due to many factors that include poor sanitation and inadequate vaccination (2). The World Health Organization (WHO) recommends three doses of tetanus vaccine at one month interval with a booster dose every 10 years to everyone above 10 years of age (4). Harmful cultural practices are particularly important here as they impact negatively on health seeking behaviours and outcome. Some of these practices include use of topical traditional concoctions or even cow dung (8), unsterilized sharp objects, aversion for mastectomy and fear of death among cancer patients from intravenous or surgical interventions. In this case, the diagnosis of the tetanus was clinical with the portal of entry being the fungating breast cancer. Her risk was increased by ignorance and harmful cultural practice. She had poor prognosticating factors at admission like short incubation period and onset period of less than 10 days and 48 hours respectively $(5,9)$. Advanced disease, low performance status, old age and possibly large dose of the pathogens may have added to the poor outcome.

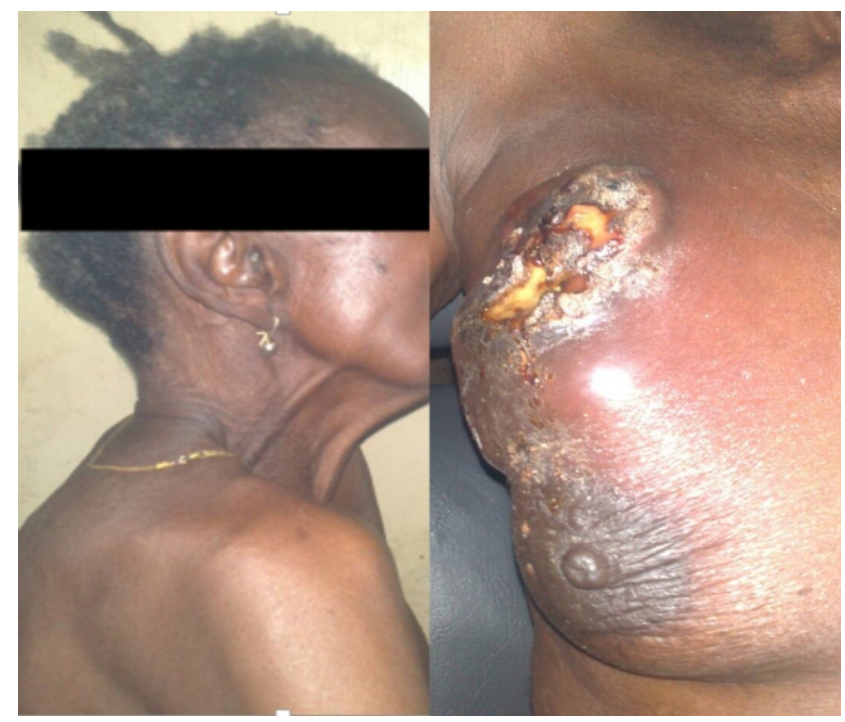

Figure 1: Neck retraction with spasm of facial and neck muscles (Left) and Fungating right breast mass with necrotic base, purulent discharge and surrounding erythema (Right)

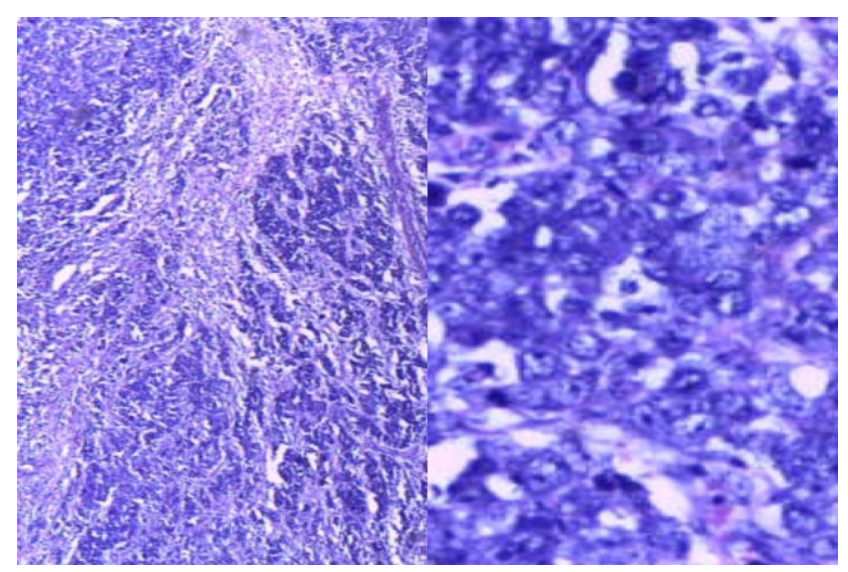

Figure 2: Haematoxlin \& Eosin (H \& E) stained showing solid sheets, nest and strands of malignant ductal cells at low power while on the right are solid sheets of malignant ductal cells, marked pleomorphism and abnormal mitotic figures. X 400

\section{Conclusion}

We presented a case of generalised tetanus complicating advanced breast cancer. Harmful cultural practices, lack of routine vaccination, late presentation, tumour characteristics and immune senescence may all be factors that predisposed the index case to tetanus infection. There is need for high index of suspicion among physicians concerning tetanus in cancer patients. There is also the need for adequate health education among the populace on cancer, vaccination and harmful cultural practices to improve health seeking behaviour and prevent tetanus.

\section{References}

1. European T, Medica E, Biobase E, Di F, Firenze C, Genova PP, et al. Lethal tetanus infection following cytotoxic chemotherapy for advanced breast cancer. European Journal of Oncology. 2015; 20(1): 5963. 
2. Toh HC, Tiam AP. Severe Tetanus in a Patient with Ulcerating Inflammatory Breast Carcinoma : A Case Report and Review of Management. Acta Onco logica (Madr). 2009; 36(1): 203.

3. Asu OT, Gever ID, Joshua NP. African Cultural Practices and Health Impli cations for Nigeria Rural Development. Int Rev Manag Bus Res [Internet]. 2013; 2(1): 17683. Available from: www.irmbjpurnal.com

4. Bautista JEC, Shu LLS, Pascual JLR, D PM. Recurrent Tetanus in Diagnosed Breast Cancer Patients of the Philippine General Hospital. Acta Med Philipp [Internet]. 2015;16. Available from: http://actamedicaphilippina.com $\mathrm{ph} /$ content/recurrent-tetanus-diagnosed-breast-cancer- patients-philippinegeneral-hospital-0Links:

5. Ogunrin OA. Tetanus A review of current concept in management. J Post graduate Med. 2009;11(1): 4661.

6. Hassel B. Tetanus: Pathophysiology, Treatment, and the Possibility of Using Botulinum Toxin against Tetanus-Induced Rigidity and Spasms. Toxins (Basel). 2013; 5: 7383

7. Anele A, Bowling M, Eckert G, Gonzalez E, Kipfer H, Sauder C. Treatment of breast cancer: Imo State, Nigeria versus Indiana, USA women Comparative analytic study. J West African Coll Surg. 2014; 4(4): 3969.

8. Bennett J, Ma C, Traverso H, Bano S, Boring J. Neonatal tetanus associated with topical umbilical ghee : covert role of cow dung. Int J Epidemiol. 1999; 24: 11725.

9. Barone AA Vaz VL, Vieira AG, et al. Clinical classification of tetanus patients. Brazilian J Med Biol Res. 2006; 39: 132937. 